\title{
DESENVOLVIMENTO DE MUDAS DE ALFACE EM DIFERENTES SUBSTRATOS E CONCENTRAÇÕES
}

\author{
Mário Euclides Pechara da Costa Jaeggi ${ }^{1}$ \\ Julio Cesar Gradice Saluci ${ }^{2}$ \\ Maxwel Rodrigues Nascimento ${ }^{3}$ \\ Rogério Rangel Rodrigues ${ }^{4}$ \\ Samuel Ferreira da Silva ${ }^{5}$ \\ Andre Oliveira Souza ${ }^{6}$ \\ Alex Justino Zacarias ${ }^{7}$ \\ Israel Martins Pereira ${ }^{8}$ \\ Rebyson Bissaco Guidinelle ${ }^{9}$ \\ Wallace Luis de Lima $^{10}$
}

\begin{abstract}
Resumo: Objetivou-se com este trabalho avaliar o desempenho de diferentes substratos e concentrações, no desenvolvimento de cultivares de alface. O experimento foi conduzido em casa de vegetação, localizada no setor de Agroecologia do Instituto Federal do Espírito Santo Campus de Alegre, ES. O delineamento utilizado foi em blocos casualizados disposto em um esquema fatorial $4 \times 3 \times 2$, sendo quatro tipos substratos (S1: Plantmax®; S2: composto orgânico; S3: húmus de minhoca; S4: borra de cana-de-açúcar), três concentrações (100\%, 75\% e 50\%) e duas cultivares de alface. As variáveis avaliadas foram: massa fresca da parte aérea, massa fresca toal e altura da parte aérea. As mudas foram avaliadas ao $21^{\circ}$ dia após semeio. Os resultados demostraram que substratos orgânicos se mostraram eficazes para o desenvolvimento das cultivares de alface, os quais podem ser utilizados em diferentes concentrações em substituição ao substrato comercial.
\end{abstract}

Palavras-chave: Lactuca sativa L.; Produção; Resíduo orgânico.

\footnotetext{
1 Departamento de Produção Vegetal/Universidade Estadual Norte Fluminense, Brasil. E-mail: mariopechara@hotmail.com.

2 Instituto Federal do Espírito Santo, Brasil. E-mail: juliosaluci@gmail.com.

3 Departamento de Produção Vegetal/Universidade Estadual Norte Fluminense, Brasil. E-mail: maxwel.rn88@gmail.com.

4 Universidade Federal de Lavras/Departamento de Engenharia, Lavras, MG. E-mail: rogeriorr7@hotmail.com.

5 Departamento de Produção Vegetal/Universidade Federal do Espírito Santo, Brasil. E-mail: samuelfd.silva@yahoo.com.br.

${ }^{6}$ Instituto Federal do Espírito Santo, Brasil. E-mail: andreolisouza@gamil.com.

7 Instituto Federal do Espírito Santo, Brasil. E-mail: alexjustino12@gmail.com.

8 Instituto Federal do Espírito Santo, Brasil. E-mail: israelmartins80@gmail.com.

9 Instituto Federal do Espírito Santo, Brasil. E-mail: rebysonguidinelle@gmail.com.

10 Instituto Federal do Espírito Santo, Brasil. E-mail: wallace@ifes.edu.br.
} 